\title{
Only SF3B1 Mutation involving K700E (And Not Other Codons), Independently Predicts Overall
}

\section{Survival in Myelodysplastic Syndromes}

Rashmi Kanagal-Shamanna, M.D., ${ }^{*}$ Guillermo Montalban-Bravo, M.D., ${ }^{2}$ Koji Sasaki, M.D., Ph.D., ${ }^{2}$ Elias Jabbour, M.D., ${ }^{2}$ Carlos Bueso-Ramos, M.D., ${ }^{1}$ Yue Wei, Ph.D., ${ }^{2}$ Kelly Chien, M.D., ${ }^{2}$ Tapan Kadia, M.D., ${ }^{2}$ Farhad Ravandi, M.D., ${ }^{2}$ Gautam Borthakur, M.D., ${ }^{2}$ Kelly A. Soltysiak, Ph.D., ${ }^{2}$ Naval Daver, M.D., ${ }^{2}$ Faezeh

Darbaniyan, Ph.D., ${ }^{3}$ Mark Routbort, M.D., ${ }^{1}$ Keyur Patel, M.D., ${ }^{1}$ L. Jeffrey Medeiros, M.D., ${ }^{1}$ Sherry Pierce, R.N., ${ }^{2}$ Hagop Kantarjian, M.D., ${ }^{2}$ and Guillermo Garcia-Manero, M.D. ${ }^{2}$

Departments of Hematopathology, ${ }^{1}$ Leukemia, ${ }^{2}$ and Biostatistics ${ }^{3}$ The University of Texas MD Anderson Cancer Center, Houston, TX

Running title: Prognostic subgroups of SF3B1 mutated MDS

\section{*Corresponding Author:}

Rashmi Kanagal-Shamanna, M.D.

Associate Professor

Department of Hematopathology

The University of Texas MD Anderson Cancer Center

1515 Holcombe Blvd, Box 072

Houston, TX 77030

Email: rkanagal@mdanderson.org

Phone: +1 (713) 745-4947

Competing Interests: The authors declare no competing interests.

Acknowledgements: This work was supported in part by grants from the Ladies Leukemia League (RKS), the National Institutes of Health, National Cancer Institute University of Texas MD Anderson Cancer Center Support award CA016672 (all authors) and the University of Texas MD Anderson MDS/AML Moon Shot (YW, KAS, FD, HK, and GG-M).

Key Words: SF3B1, ring sideroblasts, myelodysplastic syndromes, prognosis 
Abstract Word Count: 250 (250 max)

Text Word Count: 3,401 (3500 max)

References: 30 (30 max)

Tables: 4

Figures: 5

Supplemental Files: 0

Data Sharing Statement: The datasets generated during and/or analyzed during the current study are not publicly available due to patient privacy concerns but are available from the corresponding author on reasonable request.

\section{Research in Context:}

\section{Evidence before this study}

We designed this study based on the collective evidence from a systematic search of the literature for outcomes of patients MDS with SF3B1 mutations (SF3B1 ${ }^{m u t}$ ) from January 2013 to June 2020 . Both the International Working Group for the Prognosis of MDS (IWG-PM) proposal and 2016 revisions to the World Health Organization (WHO) Classification of Myelodysplastic Syndromes recognize SF3B1 ${ }^{\text {mut }}$ MDS with $<5 \%$ blasts (or ring sideroblasts $>5 \%$ for $\mathrm{WHO}$ ) as a distinct sub-category, in the absence of other unfavorable features. This was largely based on favorable prognostic outcomes, a distinct gene expression profile, and association with ring sideroblasts. However, the natural history of SF3B1 ${ }^{\text {mut }}$ MDS is heterogeneous. A high proportion of SF3B1 mutations occur within codon K700, leading to large-scale mRNA downregulation due to branch point recognition error, while the rest occur outside of this codon. The downstream functional effects of SF3B1 mutations outside of the K700 codon are unclear. The clinical course of SF3B1 $1^{\text {mut }}$ MDS patients likely depends on the type of SF3B1 mutation and other features such as variant allele frequency, concomitant gene mutations, and karyotype. Until now, the effects of the different types of SF3B1 mutations were largely unknown. 


\section{Added value of this study}

In this study, we report distinctive clinicopathologic characteristics and outcomes of MDS patients with SF3B1 mutations segregated based on mutation type: K700E vs. non-K700E. We show that $\sim 40 \%$ of SF3B1 mutated MDS patients have non-K700E mutations. Non-K700E SF3B1 ${ }^{\text {mut }}$ MDS have distinct clinico-pathologic features, such as lower ANC and frequent association with mutations in RUNX1, $B C O R, I D H 2$, and SRSF2. There was no significant difference in karyotype or SF3B1 variant allele frequency. Importantly, K700E SF3B $1^{\text {mut }}$ MDS had superior overall survival compared to $S F 3 B 1^{\text {wt }}$, in all MDS, low-grade MDS, and MDS with ring sideroblasts, but no significant difference was seen with nonK700E. By multivariate analysis, absence of $S F 3 B 1^{m u t} \mathrm{~K} 700 \mathrm{E}$, but not non-K700E, independently associated with prognosis.

\section{Implications of all the available evidence}

To the best of our knowledge, this is the first study to report these findings from a single-institutional series of MDS primarily treated with hypomethylating agents. Our study highlights the importance of determining the SF3B1 mutation type in MDS risk assessment. These findings are important in light of the recent FDA approval of luspatercept based on the results of the MEDALIST trial that suggested sustained hematological responses in SF3B1 $1^{\text {mut }}$ MDS patients. 


\section{ABSTRACT}

Background: SF3B1 mutations $\left(S F 3 B 1^{m u t}\right)$ in myelodysplastic syndromes (MDS) frequently involve codon K700E and have a favorable prognosis. The prognostic effect of non-K700E SF3B1 ${ }^{\text {mut }}$ is uncertain.

Methods: We analyzed the clinical-pathologic features and outcomes of a single-institutional series of 94 SF3B1 $1^{\text {mut }}$ and 415 SF3B1 ${ }^{\text {wt }}$ newly diagnosed untreated MDS patients and explored the differences between K700E and non-K700E subgroups.

Findings: Ninety-four (19\%) patients had SF3B1 $1^{\text {mut: }}$ median age, 74 years. Fifty-five $(60 \%)$ patients carried K700E. Recurrent non-K700E mutations (39, 40\%) included R625, H662 and K666. Compared to SF3B1 ${ }^{\text {mut }}$ K700E, non-K700E patients had a lower median ANC (1.8 vs. $\left.2 \cdot 4, p=0.005\right)$ and were frequently "high" R-IPSS (revised International Prognostic Scoring System) [7(19\%) vs. 2(4\%), p=0.031]. Non-K700E MDS frequently associated with $R U N X 1$ (26\% vs. $7 \%, \mathrm{p}=0.012)$ and exclusively with $B C O R$, $I D H 2$, and SRSF2 mutations. There was no significant difference in karyotype or SF3B1 variant allele frequency. Most $(\sim 80 \%)$ were treated with hypomethylating agents. SF3B1 ${ }^{\text {mut }}$ had superior overall survival (OS) than $S F 3 B 1^{\text {wt }}$ in all MDS categories [not-reached vs. 25.2 months, $p=0.0003$ ], low-grade MDS, and MDS with ring sideroblasts (MDS-RS). Compared to $S F 3 B 1^{\text {wt }}, S F 3 B 1^{m u t} \mathrm{~K} 700 \mathrm{E}$ had superior outcomes in all MDS categories (25 months vs. not-reached, $p=0 \cdot 0001)$, low-grade MDS, and MDS-RS, but no significant difference was seen with non-K700E. By multivariate analysis, absence of SF3B1 K700E (not non-K700E) independently associated with prognosis.

Interpretation: SF3B1 $1^{\text {mut }}$ MDS show distinct clinical and mutational profiles, with K700E showing a significantly better OS compared to non-K700E mutations and SF3B1 $1^{\text {wt }}$. Our study highlights the importance of SF3B1 mutation type in MDS risk assessment.

Funding: This work was supported by grants from the Ladies Leukemia League, the National Institutes of Health (CA016672) and the MD Anderson MDS/AML Moon Shot. 


\section{INTRODUCTION}

SF3B1 is one of the most frequently mutated genes in MDS. ${ }^{1}$ SF3B1 mutations are detected in a third of MDS patients and are present in up to $66 \%$ of MDS sub-categories with increased ring sideroblasts (RS). ${ }^{2-4}$ Prior studies have indicated that the presence of SF3B1 mutation (SF3B1 ${ }^{\text {mut }}$ ) was an independently favorable prognostic factor for survival in MDS. ${ }^{2,5-8}$ Based on the favorable outcome, a distinct gene expression profile, and association with ring sideroblasts (RS), the 2016 revisions to the World Health Organization (WHO) Classification of Myelodysplastic Syndromes recognized MDS-RS with single lineage (MDS-RS-SLD) and multilineage dysplasia (MDS-RS-MLD) as distinct sub-categories, and recommended testing for SF3B1 mutation in MDS patients with 5 and $15 \%$ ring sideroblasts in bone marrow (BM) aspirates. ${ }^{9}$ We have previously shown that the survival of MDS-RS-MLD patients (a majority of which had SF3B1 mutations) was significantly better compared to those with MDS-MLD without RS. ${ }^{10}$ Based on recent data suggesting sustained hematological responses in SF3B1 ${ }^{\text {mut }}$ MDS patients treated with luspatercept, ${ }^{11}$ the International Working Group for the Prognosis of MDS (IWG-PM) has proposed that SF3B1 ${ }^{\text {mut }}$ MDS be considered a distinct entity with a favorable prognosis in the absence of $>5 \%$ BM blasts or $\geq 1 \%$ peripheral blood (PB) blasts, absence of del(5q), monosomy 7 , inv(3), abnormal 3q26 or complex karyotype (CK), mutations in RUNX1 and EZH2, and findings suggestive of other WHO-defined entities. $^{6}$

Despite this body of information, the natural history of SF3B1 mutated MDS is heterogeneous. A high proportion of SF3B1 mutations occur within codon 700, causing a branch point recognition error of a cryptic splice site followed by nonsense decay due to aberrant splice junctions, ultimately resulting in large-scale downregulation of mRNA. ${ }^{12-14}$ The remainder of SF3B1 mutations occur outside of this hotspot and the downstream functional effects are not clear. Dalton, et al has recently suggested that SF3B1 K666N mutations had distinctive RNA splicing profiles and were associated with distinct clinico-pathologic features and worse outcomes. The authors suggested that these mutations may need aggressive management, even in the setting of a lower IPSS-R category. ${ }^{15}$ Beyond the SF3B1 mutation type, the clinical course can be potentially altered by other parameters, such as the variant allele frequency (VAF), presence of concomitant gene mutations and karyotype. 
In this study, we investigated the clinico-pathologic and genetic features and outcomes in a single-institutional series of $94 S F 3 B 1^{m u t}$ MDS patients and compared them to 415 with wild-type MDS $\left(S F 3 B 1^{\text {wt }}\right)$. We also explored the differences between the SF3B1 K700E and non-K700E mutated MDS subgroups, and demonstrated distinct clinical and mutational profiles, with the K700E mutated subgroup showing a significantly better overall survival (OS) compared to the non-K700E subgroup. Further, only the SF3B1 K700E mutation subtype independently predicted for better OS in MDS.

\section{MATERIALS AND METHODS}

\section{Patients and samples}

We selected BM aspirates from all newly diagnosed previously untreated MDS patients who underwent 81-gene panel next-generation sequencing (NGS) at The University of Texas MD Anderson Cancer Center (MDACC) from 2017-2019. Diagnoses were confirmed by BM examination and subclassified using the 2016 WHO criteria. ${ }^{16}$ At least 20 metaphases were evaluated, and interpreted according to the 2016 International System for Human Cytogenetic Nomenclature. Risk stratification was done using the IPSS-R ${ }^{17}$ for patients with MDS. The study was approved by the MDACC Institutional Review Board and all samples were collected following institutional guidelines with informed consent in accord with the Declaration of Helsinki.

\section{Targeted next-generation sequencing}

All patient samples underwent comprehensive NGS-based mutation analysis using our 81-gene panel comprising the hotspots and whole coding regions of myeloid leukemia-related genes in a CLIAcertified Molecular Diagnostics Laboratory, as previously described. ${ }^{18}$ Briefly, genomic DNA was extracted from fresh BM aspirates using standard techniques, followed by library preparation and amplicon-based targeted NGS on a MiSeq sequencer. Sequences were aligned to the GRCh37/hg19 reference genome. With a minimum of $250 \mathrm{X}$ bidirectional coverage, a minimum variant call of $2 \%$ was considered as the limit of detection. The somatic nature of the variants was inferred based on the information in online SNP databases (i.e., Exome Aggregation Consortium [ExAC], dbSNP 137/138, 1000 
Genomes) and the literature. FLT3-ITD mutations were assessed by PCR-based capillary electrophoresis.

\section{Statistical analysis}

OS was calculated as the time from diagnosis to death or last follow-up date. Patients alive at their last follow-up were censored on that date. The Kaplan-Meier product limit method was used to estimate the median OS for each clinical/demographic factor. Univariate Cox proportional hazards regression analysis was used to identify any association with each of the variables and survival outcomes followed by multivariate analysis. Response assessment was performed following 2006 IWG criteria. ${ }^{19}$

\section{Role of the funding sources}

The funding sources had no involvement in the study design; in the collection, analysis, and interpretation of data; in the writing of the report; or in the decision to submit the paper for publication.

\section{RESULTS}

\section{Patient characteristics}

A total of 509 treatment naïve MDS patients presented to our institution between 2017 and 2019, of which $94(18.5 \%)$ had SF3B1 mutations. None of the patients had received prior therapy, including erythroid stimulating agents. The clinical characteristics of patients analyzed are shown in Table 1. The SF3B1 ${ }^{\text {mut }}$ MDS patients included 59 men and 35 women, with a median age of 74 (range, 39-92) years. The distribution of IPSS-R scores for this cohort were as follows: 14 (14.9\%) very low; 37 (39.4\%) low; 13 (13.8\%) intermediate; 9 (9.6\%) high; and $12(12.8 \%)$ very high. Seventeen (18\%) patients had received prior chemotherapy and/or radiation for an unrelated malignancy (therapy-related MDS, t-MDS). Among the remaining patients, 2016 WHO sub-classifications were as follows: 22 (23\%) with MDS with single lineage dysplasia and ring sideroblasts (MDS-RS-SLD); 30 (32\%) with MDS with ring sideroblasts and multilineage dysplasia (MDS-MLD-RS); 5 (5\%) with MDS-MLD; 31 (33\%) with MDS with excess blasts; 4 (4\%) with MDS and isolated del(5q); and $2(2 \%)$ with MDS-unclassifiable (MDS-U). Treatment data was 
available for 313 patients: 248 (79\%) patients received hypomethylating agents (HMAs) alone or in combination with other agents and $35(11.2 \%)$ received chemotherapy-based regimens.

\section{Clinical and morphologic findings of SF3B1 ${ }^{m u t}$ MDS}

Compared to $S F 3 B 1^{\text {mt }}, S F 3 B 1^{\text {mut }}$ MDS patients had a significantly higher median age (74 vs. 70 , $\mathrm{p}=0.0008$ ), higher median mean corpuscular volume (MCV) (105 vs. 96, $\mathrm{p}<0.0001$ ), higher median platelet count (188 vs. $78, \mathrm{p}<0.0001$ ), and lower BM blast percentage (median, 2 vs. $4, \mathrm{p}=0.003$ ). There was no significant difference in median serum erythropoietin levels (108 vs. 57). Compared to $S F 3 B 1^{\text {wt }}$, SF3B $1^{\text {mut }}$ MDS patients were more likely to be in the IPSS-R very-low ( $4 \%$ vs. $\left.14.9 \%, p=0.0004\right)$ and low (25.5\% vs. $39.4 \%, p=0.010)$ categories and less likely to be in the IPSS-R-high $(21.5 \%$ vs. $9 \cdot 6 \%$, $\mathrm{p}=0.008)$ and very-high $(28.8 \%$ vs. $12.8 \%, \mathrm{p}=0.001)$ categories. The median percentage of ring sideroblasts was higher in patients with SF3B1 ${ }^{m u t}$ MDS (40\% vs. $\left.7 \%, p<0.0001\right)$ compared to the those with SF3B1 ${ }^{\text {wt }}$; accordingly, the SF3B1 ${ }^{\text {mut }}$ MDS group was significantly enriched in WHO categories with ring sideroblasts [MDS-SLD-RS (23.4\% vs. $1.2 \%, \mathrm{p}<0.0001)$ and MDS-RS-MLD $(31.9 \%$ vs. $6 \cdot 7 \%$, $\mathrm{p}<0.0001)$ ] and MDS with isolated del(5q) (4.3\% vs. 0.7\%, $\mathrm{p}<0.0001)$, while they were underrepresented in MDS-MLD (5.3\% vs. $33 \%, p<0.0001)$, MDS-EB (33\% vs. $48 \cdot 4 \%, p<0.0001)$, and MDS-U (2.1\% vs. $9 \cdot 9 \%, p=0 \cdot 012)$.

SF3B1 $1^{\text {mut }}$ MDS patients were less likely to be therapy-related [17 (18\%) vs. $\left.142(34 \%) ; p=0 \cdot 002\right]$. However, within 24 t-MDS with $>15 \%$ RS and $<5 \%$ blasts, the most frequent mutation observed was in SF3B1 $(n=13,54 \%), 4$ of which had concurrent TP53 mutations/CK. Six (25\%) patients had TP53 mutations/CK without SF3B1 mutations, and 5 (20.8\%) had neither SF3B1 nor TP53 mutations/CK.

\section{Mutational landscape of SF3B1 ${ }^{\text {mut }}$ MDS}

BM aspirates from all patients underwent NGS analysis with an 81-gene panel at the time of diagnosis. The most frequent SF3B1 mutation, noted in $\sim 60 \%$ of all patients, was the hotspot K700E. Among the remaining mutations observed, the most frequent involved the codons H662, K666, and R625, seen in 8 patients each (Figure 1 A). The mutational landscape is depicted in Figure 1 B. Only a third of the patients had an SF3B1 mutation as the sole driver of MDS, while the majority had concomitant 
mutations. The genes mutated in $>10 \%$ of patients in decreasing order of frequency included TET2 (25\%), DNMT3A (21\%), RUNX1 (15\%), TP53 (10\%), ASXL1 (7\%), BCOR (4\%), IDH1/2 (4\%), SRSF2 (3\%), NRAS (3\%), and EZH2 (3\%). Conventional cytogenetic analysis showed a higher frequency of normal karyotype and lower frequency of $C K$ in $S F 3 B 1^{m u t}$ compared to $S F 3 B 1^{\text {wt }}$ cases. Three $S F 3 B 1^{\text {mut }}$ patients had EVI1 gene rearrangements, but the frequency was not significantly different compared to the SF3B1 ${ }^{\text {wt }}$ group. Three SF3B1 ${ }^{\text {mut }}$ patients had $t(1 ; 3)(p 36 ; q 21)$ RPN1/PRDM16, which was absent in the SF3B $1^{\text {wt }}$ cohort.

\section{Comparison between SF3B1 K700E and non-K700E mutated MDS}

We compared the clinico-pathologic features of 55 K700E vs. 39 non-K700E treatment naïve SF3B1 ${ }^{\text {mut }}$ MDS patients (Table 2). MDS with SF3B1 K700E mutations had a higher percentage of ring sideroblasts (median $50 \%$ vs. $34 \%$; $=0.038$ ), higher ANC ( 2.4 vs. $1.8, p=0.005$ ), and a trend of higher platelet count (196 vs. 124, p=0.05). Among IPSS-R categories, SF3B1 ${ }^{\text {mut }}$ MDS patients with non-K700E mutations had a significantly higher representation in IPSS-R high category [7(19\%) vs. 2(4\%), $p=0.031$ ], but the distribution was not significantly different among other categories. Likewise, within 2016 WHO categories, SF3B1 $1^{\text {mut }}$ MDS patients with K700E mutations were less likely to be classified as MDS-EB than non-K700E SF3B1 mutated patients (22\% vs. $49 \%, p=0.008)$. All 4 patients that fit the criteria for MDS with isolated del(5q) had K700E mutations.

There was no difference in the median SF3B1 variant allele frequency (VAF) between the 2 groups. The frequency of $R U N X 1$ mutation was significantly higher in non-K700E cases (26\% vs. $7 \cdot 3 \%$, $\mathrm{p}=0.012)$, and mutations in BCOR $(\mathrm{p}=0.02), I D H 2(\mathrm{p}=0.07)$, and SRSF2 $(\mathrm{p}=0.07)$ were exclusively observed in non-K700E cases (Figure 2). No significant differences were noted in the frequencies of TP53 or clonal hematopoiesis-associated mutations, including DNMT3A, ASXL1, and TET2.

There were no significant differences in normal vs. complex karyotype. When cytogenetic aberrations were classified using the comprehensive cytogenetic scoring system (CCSS; scores from 05), SF3B1 ${ }^{\text {mut }}$ K700E mutated patients were more likely to have a lower CCSS scores (0/1) compared to non-K700E MDS patients [39 (80\%) vs. 19 (53\%); $p=0 \cdot 011]$. 


\section{Therapy and outcomes}

The majority of patients were treated with an HMA: 16/17 (94\%) K700E mutated patients; 15/19 (79\%) non-K700E mutated patients; and 217/277 (78\%) SF3B1 ${ }^{\text {wt }}$ patients. With a median follow-up of 15.6 months, SF3B1 ${ }^{\text {mut }}$ MDS patients had a significantly better OS than $S F 3 B 1^{\text {wt }}$ patients. This was true in the entire cohort (not reached vs. 25.2 months, $p=0.0003$ ), low-grade MDS categories (not reached vs. $41 \cdot 3$ months, $p=0 \cdot 0015$ ), and low-grade MDS-RS categories (not reached vs. $22 \cdot 3$ months, $p=0 \cdot 0004$ ). When segregated based on SF3B1 mutation types, 4 (7.3\%) of the K700E mutated patients died, while 9 (23\%; $\mathrm{p}=0.036$ ) of non-K700E mutated patients died. The outcomes of K700E SF3B1 ${ }^{\text {mut }}$ mutated MDS patients were significantly better compared to $S F 3 B 1^{\text {wt }}$ within the entire cohort (median OS, not reached vs. 25.2 months, $\mathrm{p}=0.0001$ ), and within the low-grade MDS (median OS, not reached vs. 41.3 months, $\mathrm{p}=0.0015$ ) and MDS-RS categories (median OS, not reached vs. 22.3 months, $p=0.0001$ ). In contrast, the outcomes of non-K700E SF3B $1^{\text {mut }}$ MDS patients were similar to SF3B1 $1^{\text {wt }}$ MDS patients, within the entire cohort (median OS, not reached vs. 25.2 months, $p=0.2314$ ), and within the low-grade MDS (median OS, not reached vs. 41.3 months, $\mathrm{p}=0.2598$ ), and MDS-RS categories (median OS, not reached vs. $22 \cdot 3$ months, $p=0.2327)$. Within the MDS-EB categories, compared to $S F 3 B 1^{\text {wt }}$, there were no significant differences in OS of K700E SF3B1 ${ }^{\text {mut }}$ (median OS, not reached vs. 17.7 month, $\mathrm{p}=0.355$ ) and non-K700E SF3B1 ${ }^{\text {mut }}$ (median OS, 20.5 vs. 17.7 months; $p=0.5477$ ) patients (Figure 3A-D).

Within the entire MDS cohort, by univariate analysis (Table 3), the following parameters were associated with worse outcomes: higher BM blasts percentage; lower hemoglobin, platelet and MCV; prior history of chemo-radiation (t-MDS); presence of CK; higher IPSS-R risk category; WHO MDS-EB category; absence of mutations in SF3B1 K700E, TET2, and U2AF1; and presence of a TP53 mutation. The outcome was superior when the SF3B1 mutation VAF was higher [ $\mathrm{p}=0.026$; OR, 0.96 (0.924-0.995)]. Non-K700E SF3B1 mutations did not associate with OS. By multivariable analysis (using a p-value cut-off of 0.200 ), lower hemoglobin, higher IPSS-R category, absence of SF3B1 K700E, and presence of TP53 mutation were independent predictors of worse OS. Within the MDS-RS categories (MDS-RS-SLD and MDS-RS-MLD), independent prognostic factors of worse OS included lower platelet count and presence of mutations in SF3B1 (non-K700E), ASXL1, SRSF2, and TP53 (Table 4). 
Given the known independent prognostic influence of both SF3B1 and TP53 mutations, we assessed the co-mutation pattern and outcomes of SF3B1 mutations with TP53 mutations and CK. A total of 14 SF3B1 ${ }^{\text {mut }}$ patients had concurrent TP53 mutations [ $(\mathrm{n}=9$; median VAF $25 \cdot 8$ (range, 2-68\%)] or CK $(n=5)$. We divided the patients into 4 categories: (1) SF3B1 ${ }^{\text {wt }}$ MDS without TP53 ${ }^{\text {mut }}$ or CK $(n=219)$; (2) SF3B1 ${ }^{\text {mut }}$ MDS without TP53 ${ }^{\text {mut }}$ or CK $(\mathrm{n}=71)$; (3) SF3B1 $1^{\text {mut }}$ MDS with TP53 ${ }^{\text {mut }}$ or CK $(\mathrm{n}=14)$; and (4) SF3B1 ${ }^{\text {wt }}$ MDS with TP53 ${ }^{\text {mut }}$ or CK $(\mathrm{n}=153)$. No survival differences were noted between patients with SF3B1 ${ }^{\text {mut }}$ MDS with or without mutated TP53 and/or CK (median OS, not reached) and patients with SF3B $1^{\text {wt }}$ MDS without TP53 mutations/CK (44.3 months). But MDS patients with TP53 mutations or CK had a significantly worse outcomes (median OS, 12.9 months, HR 1.46, p=0.001) in the absence of SF3B1 mutations. Similar findings were noted within the low-grade MDS and MDS-RS categories, where worse OS was associated with TP53 mutations or CK when SF3B1 was wild-type. This suggests that SF3B1 mutation may negate the poor prognostic effect of TP53 mutation or CK (Figure 4).

\section{SF3B1 mutation acquired during disease course}

SF3B1 mutation is considered a founder clone, however we observed 2 patients in which the mutation arose during disease evolution. The first patient was a 74 year old man who was diagnosed with MDS-EB with trisomy 8 and mutations in BCOR, DNMT3A (x2), EZH2, TET2, and U2AF1 (33\% VAF). BM morphology did not show any ring sideroblasts. Flow cytometry analysis showed $5 \%$ B-cells with a CLL/SLL-like immunophenotype. Following HMA treatment, a 6-month follow-up BM aspirate analysis showed persistent MDS with $2 \%$ blasts and clonal evolution with additional sub-clonal mutations in TET2 and SF3B1 R625C (VAF, 29.6\%), expansion in the EZH2 clone, clearance of BCOR, and reduction in previously detected TET2 and U2AF1 mutations. As expected, given the acquisition of the SF3B1 mutation, BM morphology showed 18\% ring sideroblasts (Figure 5A). The second case was a 68 year old woman with pancytopenia. BM aspirate analysis showed MDS-EB with multi-lineage dysplasia and MF-3 fibrosis with an del(5q) abnormality in 16 of 20 metaphases. NGS analysis demonstrated a JAK2 V617 $(<5 \%$ VAF) mutation at baseline. The patient underwent treatment with an HMA and PD-1/CTLA-4 blockers, and transformed to AML over the next 4 months. Karyotype showed del(5q) abnormality. Additional mutations in SF3B1 K700E (29\% VAF) and SETBP1 E862K (23.8\% VAF) were acquired at 
transformation (Figure 5B). Both the patients underwent allogenic stem cell transplantation, and have remained in morphologic and molecular remission.

\section{DISCUSSION}

The outcomes of $S F 3 B 1^{m u t}$ MDS, although regarded as a favorable prognostic biomarker, are variable. In this study, to understand the heterogeneity in clinico-pathologic features and outcomes of different types of SF3B1 mutations, we reviewed the entire spectrum of MDS, including low-grade $(<5 \%$ blasts) through high-grade ( $\geq 5 \%$ blasts) MDS.

SF3B1 mutations are observed in a third of MDS cases, are associated with $\geq 15 \% \mathrm{RS}$, and are overrepresented in the MDS-RS-SLD and MDS-RS-MLD WHO subtypes. ${ }^{2,3,20}$ Ring sideroblasts represent dysplastic erythroid precursors with abnormal iron-laden mitochondria encircling the nucleus. ${ }^{21,22}$ Based on the distinct gene expression profile of $S F 3 B 1^{m u t}$ MDS and strong association with RS, the current WHO criteria classifies MDS with <5\% BM blasts and 5-15\% RS in the absence of isolated de(5q) with SF3B1 mutation as equivalent to MDS-RS-SLD or MDS-RS-MLD characterized by $\geq 15 \% \mathrm{RS}$. $^{9}$ SF3B1 mutation is regarded as the only favorable prognostic biomarker in MDS. ${ }^{2}$ The most frequent hotspot SF3B1 mutation is located on K700E, while the rest are located within exons 14-16.,4,14

Consistent with the literature, our study found that SF3B1 $1^{\text {mut }}$ MDS had favorable clinical characteristics, with enrichment in lower IPSS-R categories and ring sideroblastic WHO subtypes. However, when segregated by the type of mutation, we demonstrated that only a subset of $S F 3 B 1^{\text {mut }}$ MDS patients, those with K700E mutations, had favorable outcomes, while the outcomes of those with non-K700E mutations, noted in $\sim 40 \%$ of all SF3B1 mutated patients, were similar to those with SF3B1 ${ }^{\text {wt }}$ MDS. The only significant adverse clinical feature in non-K700E mutated SF3B1 was lower ANC. Both K700E and non-K700E SF3B1 ${ }^{\text {mut }}$ MDS patient groups showed frequent RS, with a median RS percentage exceeding $15 \%$ in both. Despite no significant difference in median BM blast percentage, these cases had a higher representation in the MDS-EB categories and a higher frequency of IPSS-R high cases. There were no karyotypic differences, but non-K700E SF3B1 ${ }^{m u t}$ MDS had a higher frequency of concomitant mutations in RUNX1 (>25\% cases) and BCOR. RUNX1 mutation is an independent predictor of poor survival in MDS. ${ }^{6,23}$ Presence of K700E SF3B1 ${ }^{\text {mut }}$ alone (not non-K700E mutations) was associated with 
favorable OS in both univariate and multivariate analysis, along with other prognostic factors including hemoglobin, IPSS-R category, and TP53 mutations. The latter finding was true within low-grade MDS and MDS-RS categories. Thus, recognition of SF3B1 mutation type by sequencing the entire coding region is important and has bearing on clinical management. Hence, incorporation of the type of SF3B1 mutation rather than simply the presence of mutation and ring sideroblast percentage will be helpful for future WHO sub-classification.

SF3B1 mutation is an MDS initiating clone arising in hematopoietic stem cells of lymphomyeloid origin, with the mutation often being the sole driver with a near-heterozygous VAF. ${ }^{24,25}$ Prior studies demonstrated that the presence of SF3B1 mutation, in the context of clonal hematopoiesis or clonal cytopenia(s), was an independent predictor of progression to MDS, unlike isolated mutations in DNMT3A or TET2. ${ }^{26}$ Within our MDS cohort, $>20 \%$ of $S F 3 B 1^{m u t}$ MDS patients had common CHIP-associated gene mutations in TET2 and DNMT3A. The presence of these additional gene mutations was not associated with multi-lineage dysplasia. TP53 mutations were noted in $>10 \%$ of SF3B ${ }^{\text {mut }}$ MDS patients. Since TP53 mutations associated with CK, as observed in $\sim 10 \%$ of MDS patients, we assessed the interplay of these 3 parameters on outcomes. Our findings showed that SF3B1 mutations negate the poor prognostic effects of TP53 mutations and/or CK. SF3B1 mutation was less frequent within t-MDS, but was more frequent than TP53 mutations within t-MDS with RS.

In addition to $\mathrm{CK}$, other poor prognostic cytogenetic features in a subset of $S F 3 B 1^{\text {mut }}$ MDS included del(7q)/-7 and EVI1/MECOM rearrangement. Studies have shown enrichment of SF3B1 mutations in $\mathrm{AML}$ with $\operatorname{inv}(3)$ or $\mathrm{t}(3 ; 3)$ and overexpression of $E V I 1$ in $S F 3 B 1^{\text {mut }}$ MDS that transform to $\mathrm{AML}^{27,28}$ In our cohort, the frequency of EVI1 rearrangement was not significantly different from the wildtype cases. There were also $3 S F 3 B 1^{m u t}$ patients with $t(1 ; 3)(p 36 ; q 21)$, a rare but recurrent rearrangement in MDS/AML involving PRDM16 (PR/SET domain containing 16) or MEL1 (located on 1p36) and the enhancer element of GATA2 (RPN1), located on 3q21. PRDM16 is zinc finger transcriptional regulator structurally similar to MECOM (MDS1 and EVI1 complex). Rearrangements of PRDM16 in $\mathrm{t}(1 ; 3)(\mathrm{p} 36 ; \mathrm{q} 21)$ and MECOM in inv(3)(q21q26.2) or $\mathrm{t}(3 ; 3)(\mathrm{q} 21 ; \mathrm{q} 26.2)$ to the GATA2/RPN1 locus lead to overexpression of the aberrant oncogenic short-forms of PRDM16s and EVI1 that lack the PR/SET domain. Aberrant 
Prdm16s has been implicated in leukemogenesis or progression by transformation of megakaryocyteerythroid progenitors to myeloid leukemia stem cells. ${ }^{29,30}$

Within this single institution large dataset, we were able to evaluate SF3B1 mutations in unique clinical scenarios, including isolated del(5q) (all of which were associated with K700E mutations), and tMDS. A high proportion of cases with TP53 mutation or CK (54\%) were associated with t-MDS. Within SF3B1 ${ }^{\text {mut }}$ MDS, a high proportion of t-MDS cases had concurrent TP53 mutations and/or CK (57\%), while fewer had SF3B1 mutations without a TP53 mutation or CK (19\%). There were no differences in outcome based on therapy-related disease [HR 2.11, 95\% Cl 0.78-5.66, $p=0 \cdot 140]$.

Our study is a retrospective analysis of a large single-institutional series. Validation of these findings in an independent cohort, as well as prospective analysis of the outcome differences between K700E and non-K700E SF3B1 ${ }^{\text {mut }}$ MDS patients, in the context of newer therapies such as luspatercept, is needed.

In summary, we show that $\sim 40 \%$ of $S F 3 B 1^{\text {mut }}$ MDS show non-K700E mutations. Hotspot K700E and non-K700E SF3B1 ${ }^{\text {mut }}$ MDS show distinct clinical and mutational profiles, with K700E showing a significantly better OS compared to non-K700E and SF3B1 $1^{\text {wt }}$. Only absence of $S F 3 B 1^{\text {mut }}$ K700E mutation independently predicted for worse OS in MDS. Hence, identification of the SF3B1 mutation type is important for risk stratification.

\section{Author Contributions:}

RK-S: Concept and design; administrative support; provision of study materials and patients; data collection, analysis, interpretation; and manuscript writing and final approval. GM-B, KS, EJ, CB-R, YW, ND, FD, MR, KP, LJM, HK, GG-M: Collection and assembly of data; data analysis and interpretation; manuscript writing; and final approval of manuscript. SP: Provision of study materials; data collection, and final approval. KAS: Administrative support; data analysis and interpretation; and manuscript writing and final approval.

\section{Author Disclosures:}

Rashmi Kanagal-Shamanna: This author declares no conflict of interest. 
Guillermo Montalban-Bravo: This author declares no conflict of interest.

Koji Sasaki: This author declares an advisory role with Pfizer Japan.

Elias Jabbour: This author declares research support and an advisory role with Adaptive, AbbVie, Amgen, Pfizer, Cyclacel LTD, Takeda, Bristol Myers Squibb.

Carlos Bueso-Ramos: This author declares no conflict of interest.

Sherry Pierce: This author declares no conflict of interest.

Yue Wei: This author declares no conflict of interest

Kelly A. Soltysiak: This author declares no conflict of interest.

Naval Daver: This author declares no conflict of interest.

Faezeh Darbaniyan: This author declares no conflict of interest.

Mark Routbort: This author declares no conflict of interest.

Keyur Patel: This author declares no conflict of interest.

L. Jeffrey Medeiros: This author declares no conflict of interest.

Hagop Kantarjian: This author declares research support and an advisory role with Actinium, and research support from AbbVie, Agio, Amgen, Ariad, Astex, BMS, Cyclacel, Daiichi-Sankyo, Immunogen, Jazz Pharma, Novartis, and Pfizer.

Guillermo Garcia-Manero: This author declares research support and an advisory role with Bristol Myers Squibb, Astex, and Helsinn, and research support from Amphivena, Novartis, AbbVie, H3 Biomedicine, Onconova, and Merck. 


\section{REFERENCES}

1 Papaemmanuil, E. et al. Clinical and biological implications of driver mutations in myelodysplastic syndromes. Blood, The Journal of the American Society of Hematology 122, 3616-3627 (2013).

2 Malcovati, L. et al. SF3B1 mutation identifies a distinct subset of myelodysplastic syndrome with ring sideroblasts. Blood 126, 233-241, doi:10.1182/blood-2015-03-633537 (2015).

3 Papaemmanuil, E. et al. Somatic SF3B1 mutation in myelodysplasia with ring sideroblasts. New England Journal of Medicine 365, 1384-1395 (2011).

4 Yoshida, K. et al. Frequent pathway mutations of splicing machinery in myelodysplasia. Nature 478, 64-69 (2011).

5 Chen, J. et al. Myelodysplastic syndrome progression to acute myeloid leukemia at the stem cell level. Nat Med 25, 103-110, doi:10.1038/s41591-018-0267-4 (2019).

6 Malcovati, L. et al. SF3B1-mutant myelodysplastic syndrome as a distinct disease subtype-A Proposal of the International Working Group for the Prognosis of Myelodysplastic Syndromes (IWG-PM). Blood (2020).

7 Migdady, Y. et al. Clinical outcomes with ring sideroblasts and SF3B1 mutations in myelodysplastic syndromes: MDS clinical research consortium analysis. Clinical Lymphoma Myeloma and Leukemia 18, 528-532 (2018).

8 Montalban-Bravo, G. \& Garcia-Manero, G. Myelodysplastic syndromes: 2018 update on diagnosis, risk-stratification and management. American journal of hematology 93, 129-147 (2018).

9 Arber, D. A. et al. The 2016 revision to the World Health Organization classification of myeloid neoplasms and acute leukemia. Blood 127, 2391-2405, doi:10.1182/blood-2016-03-643544 (2016).

10 Kanagal-Shamanna, R. et al. Validation of the 2016 revisions to the WHO classification in lowerrisk myelodysplastic syndrome. Am J Hemato/ 92, E168-E171, doi:10.1002/ajh.24776 (2017).

11 Fenaux, P. et al. Luspatercept in Patients with Lower-Risk Myelodysplastic Syndromes. New England Journal of Medicine 382, 140-151 (2020). 
12 Alsafadi, S. et al. Cancer-associated SF3B1 mutations affect alternative splicing by promoting alternative branchpoint usage. Nature communications 7, 1-12 (2016).

13 Darman, R. B. et al. Cancer-Associated SF3B1 Hotspot Mutations Induce Cryptic 3' Splice Site Selection through Use of a Different Branch Point. Cell Rep 13, 1033-1045, doi:10.1016/j.celrep.2015.09.053 (2015).

14 Obeng, E. A. et al. Physiologic expression of Sf3b1K700E causes impaired erythropoiesis, aberrant splicing, and sensitivity to therapeutic spliceosome modulation. Cancer cell 30, 404-417 (2016).

15 Dalton, W. B. et al. The K666N mutation in SF3B1 is associated with increased progression of MDS and distinct RNA splicing. Blood advances 4, 1192 (2020).

16 Arber, D. A. et al. The 2016 revision to the World Health Organization (WHO) classification of myeloid neoplasms and acute leukemia. Blood, doi:10.1182/blood-2016-03-643544 (2016).

17 Greenberg, P. L. et al. Revised international prognostic scoring system for myelodysplastic syndromes. Blood 120, 2454-2465, doi:10.1182/blood-2012-03-420489 (2012).

18 Kanagal-Shamanna, R. et al. Myeloid neoplasms with isolated isochromosome 17q demonstrate a high frequency of mutations in SETBP1, SRSF2, ASXL1 and NRAS. Oncotarget 7, 14251 (2016).

19 Cheson, B. D. et al. Clinical application and proposal for modification of the International Working Group (IWG) response criteria in myelodysplasia. Blood 108, 419-425, doi:10.1182/blood-200510-4149 (2006).

20 Malcovati, L. \& Cazzola, M. Recent advances in the understanding of myelodysplastic syndromes with ring sideroblasts. Br J Haematol 174, 847-858, doi:10.1111/bjh.14215 (2016).

21 Della Porta, M. G. et al. Minimal morphological criteria for defining bone marrow dysplasia: a basis for clinical implementation of WHO classification of myelodysplastic syndromes. Leukemia 29, 66-75, doi:10.1038/leu.2014.161 (2015).

22 Cazzola, M. \& Invernizzi, R. (Haematologica, 2011).

23 Bejar, R. et al. Clinical effect of point mutations in myelodysplastic syndromes. New England Journal of Medicine 364, 2496-2506 (2011). 
24 Mortera-Blanco, T. et al. SF3B1-initiating mutations in MDS-RSs target lymphomyeloid hematopoietic stem cells. Blood 130, 881-890 (2017).

25 Mian, S. A. et al. SF3B1 mutant MDS-initiating cells may arise from the haematopoietic stem cell compartment. Nature communications 6, 1-14 (2015).

26 Malcovati, L. et al. Clinical significance of somatic mutation in unexplained blood cytopenia. Blood 129, 3371-3378 (2017).

27 Shiozawa, Y. et al. Gene expression and risk of leukemic transformation in myelodysplasia. Blood, The Journal of the American Society of Hematology 130, 2642-2653 (2017).

28 Papaemmanuil, E. et al. Genomic classification and prognosis in acute myeloid leukemia. New England Journal of Medicine 374, 2209-2221 (2016).

$29 \mathrm{Hu}, \mathrm{T}$. et al. PRDM16s transforms megakaryocyte-erythroid progenitors into myeloid leukemiainitiating cells. blood 134, 614-625 (2019).

30 Mochizuki, N. et al. A novel gene, MEL1, mapped to 1p36. 3 is highly homologous to the MDS1/EVl1 gene and is transcriptionally activated in $t(1 ; 3)(p 36 ; q 21)$-positive leukemia cells. Blood, The Journal of the American Society of Hematology 96, 3209-3214 (2000). 


\section{FIGURE LEGENDS}

Figure 1. Mutational characteristics. (A) Spectrum of SF3B1 mutations within the coding region of the gene seen in the study group generated using MutationMapper from cbioportal. About $\sim 60 \%$ of mutations involved the hotspot K700E, while $\sim 40 \%$ involved the non-K700E regions. (B) Mutational landscape of SF3B1 K700E mutated MDS. Comutation plot of 94 SF3B1 mutated MDS patients showing nonsynonymous gene mutations (left), separated by the mutation subtype: K700E (blue) and non-K700E (yellow). Each column represents a patient, and mutations are represented by color. The frequency of gene mutations is shown on the right. Color code: Maroon indicates mutation while blue indicates wildtype. Variant allele frequency of SF3B1 is represented as a color gradient.

Figure 2. Within SF3B1 mutated MDS, the K700E mutant group has distinct clinico-pathologic and genetic characteristics compared to non-K700E mutant MDS. SF3B1 non-K700E mutated patients (red) had a significantly higher frequency of mutations in $R U N X 1$, and a trend to higher frequencies of mutations in SRSF2 and IDH2 genes compared to SF3B1 K700E mutated MDS patients.

Figure 3. Overall survival (OS) of SF3B1 mutated MDS subtypes compared to SF3B1 wild-type MDS patients. OS of SF3B1 mutated MDS patients was significantly better than wild-type patients. When segregated based on SF3B1 mutation types, the outcome of SF3B1 non-K700E patients was similar to wild-type MDS, within the entire cohort ( $A$, median $O S$, not reached for both; $p=0.02$ ), within low-grade MDS (B), and MDS-RS (C) categories, while there were no significant differences in OS within MDS-EB categories (D). The outcome of SF3B1 K700E patients was superior in all the categories. Kaplan-Meier curves show patients with SF3B1 K700E MDS mutations (red), SF3B1 non-K700E mutation MDS patients (green), and SF3B1 wild-type MDS patients (blue).

Figure 4. Survival differences in MDS based on concurrent SF3B1 and TP53 mutations and/or complex karyotype (CK). No overall survival differences were noted between SF3B1 mutated MDS patients with or without mutated TP53 and/or CK (median OS not reached) and SF3B1 wild-type MDS patients without TP53 mutations/CK (44.3 months). MDS patients with TP53 mutations or CK had significantly worse 
outcomes (median OS 12.9 months, HR 1.46, $\mathrm{p}=0.001$ ) in the absence of SF3B1 mutations, suggesting that SF3B1 mutation may negate the poor prognostic effects of TP53 mutation or CK.

Figure 5. NGS analysis of serial of samples in two MDS patients who acquired SF3B1 mutations over the course of the disease: in patient \#1, at the time of $A M L$ progression $(A)$, and in patient \#2, post HMA therapy at the time of morphologically persistent disease with genotype change corresponding to phenotypic acquisition of ring sideroblasts (B). Representative FISH plots from both cases showing clonal architecture at different time points. In both cases, mutations cleared following allogeneic stem cell transplantation. 

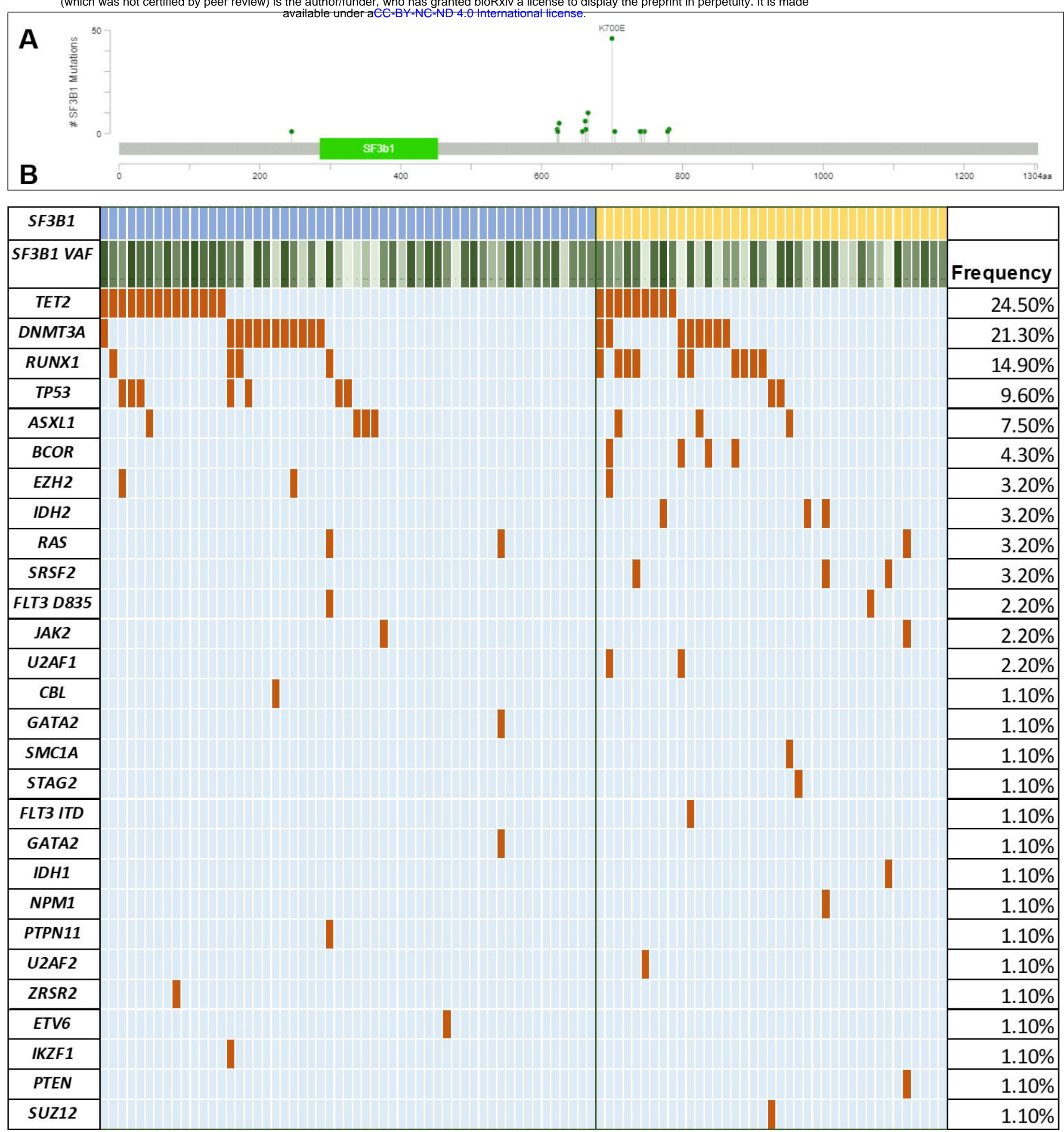


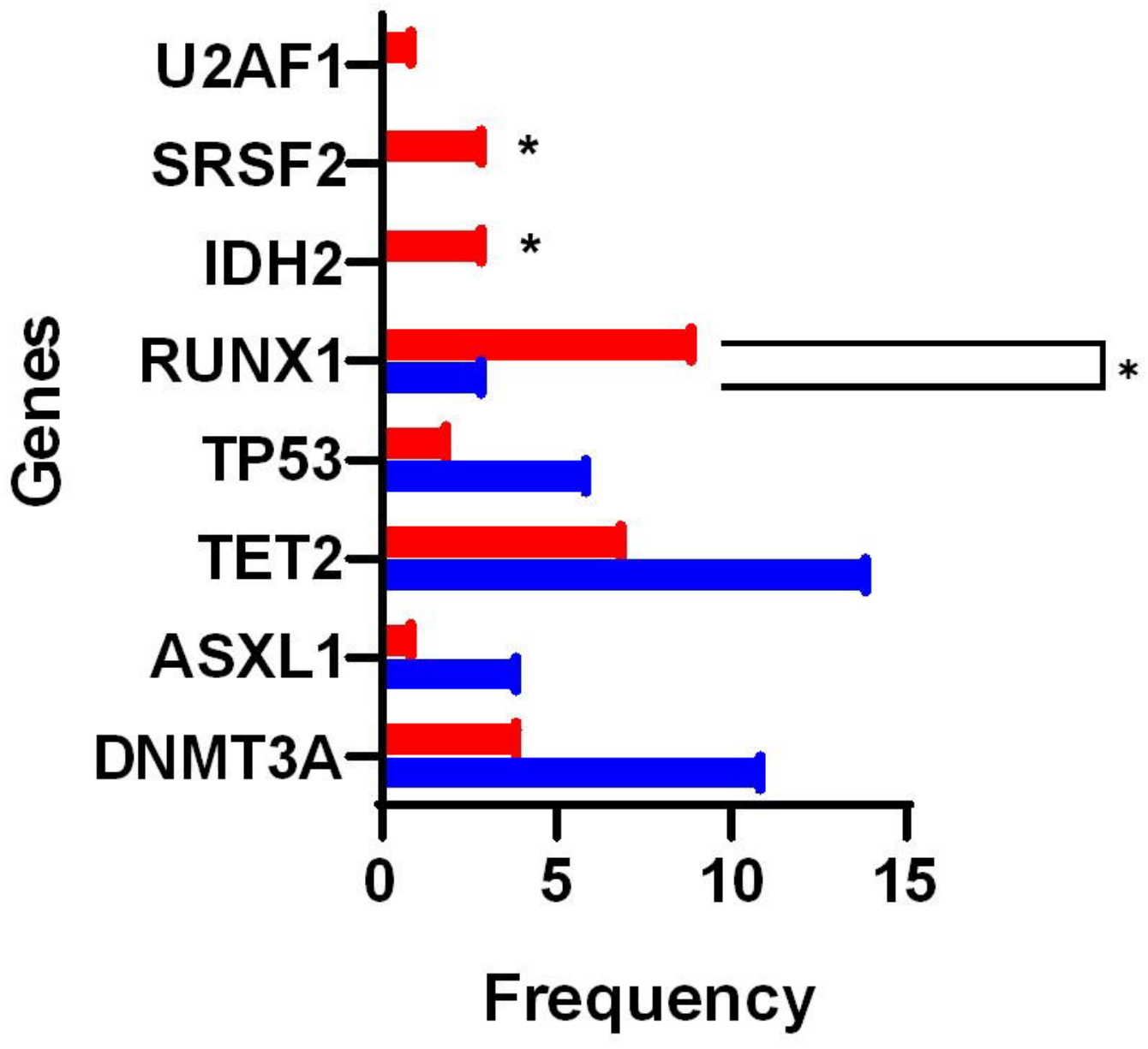




\section{All MDS}

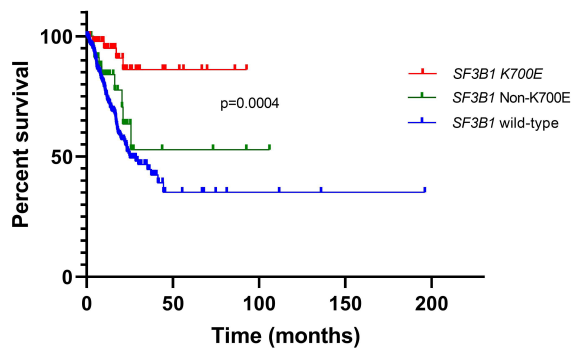

\begin{tabular}{|c|c|c|c|c|c|}
\hline All MDS & Median OS & $\mathbf{n}$ & HR & $\mathbf{9 5 \%}$ Cl & $\mathbf{p}$ \\
\hline SF3B1 K700E & NR & 54 & 0.3886 & $0.2401-0.6291$ & 0.0001 \\
\hline SF3B1 non-K700E & NR & 38 & 0.7061 & $0.3994-1.248$ & 0.2314 \\
\hline SF3B1 WT & 25.17 & 415 & - & - & - \\
\hline
\end{tabular}

MDS-RS

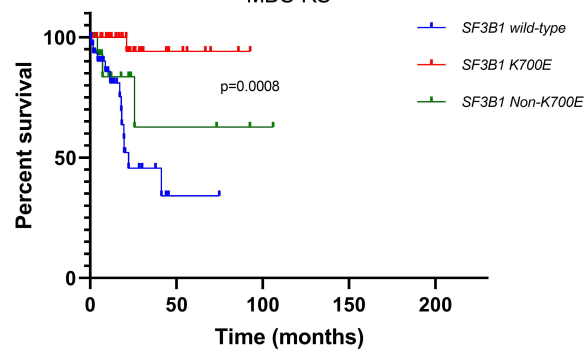

\begin{tabular}{|c|c|c|c|c|c|}
\hline MDS-RS & Median OS & $\mathbf{n}$ & HR & $\mathbf{9 5 \%}$ Cl & p \\
\hline SF3B1 K700E & NR & 35 & 0.1116 & $0.03660-0.3404$ & 0.0001 \\
\hline SF3B1 non-K700E & NR & 16 & 0.5222 & $0.1796-1.518$ & 0.2327 \\
\hline SF3B1 WT & 22.27 & 33 & - & - & - \\
\hline
\end{tabular}

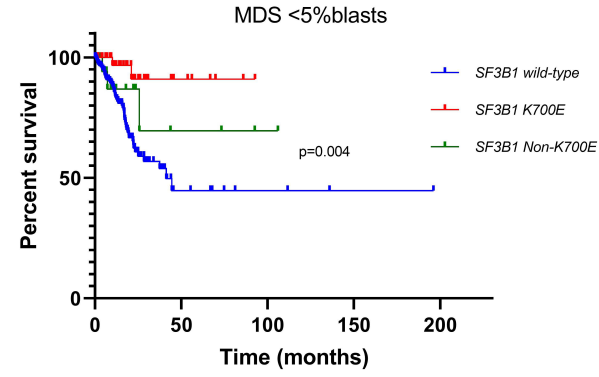

\begin{tabular}{|c|c|c|c|c|c|}
\hline MDS <5\% blasts & Median OS & $\mathbf{n}$ & HR & $\mathbf{9 5 \%}$ CI & p \\
\hline SF3B1 K700E & NR & 43 & 0.3464 & $0.1801-0.6663$ & 0.0015 \\
\hline SF3B1 non-K700E & NR & 19 & 0.6009 & $0.2477-1.457$ & 0.2598 \\
\hline SF3B1 WT & 41.33 & 214 & - & - & - \\
\hline
\end{tabular}

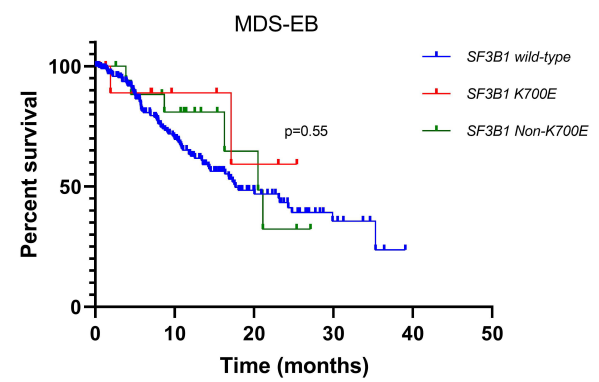

\begin{tabular}{|c|c|c|c|c|c|}
\hline MDS-EB & Median OS & $\mathbf{n}$ & HR & $\mathbf{9 5 \%} \mathbf{C l}$ & $\mathbf{p}$ \\
\hline SF3B1 K700E & NR & 11 & 0.6125 & $0.2167-1.731$ & 0.355 \\
\hline SF3B1 non-K700E & 20.5 & 19 & 0.795 & $0.3763-1.679$ & 0.5477 \\
\hline SF3B1 WT & 17.67 & 201 & - & - & - \\
\hline
\end{tabular}




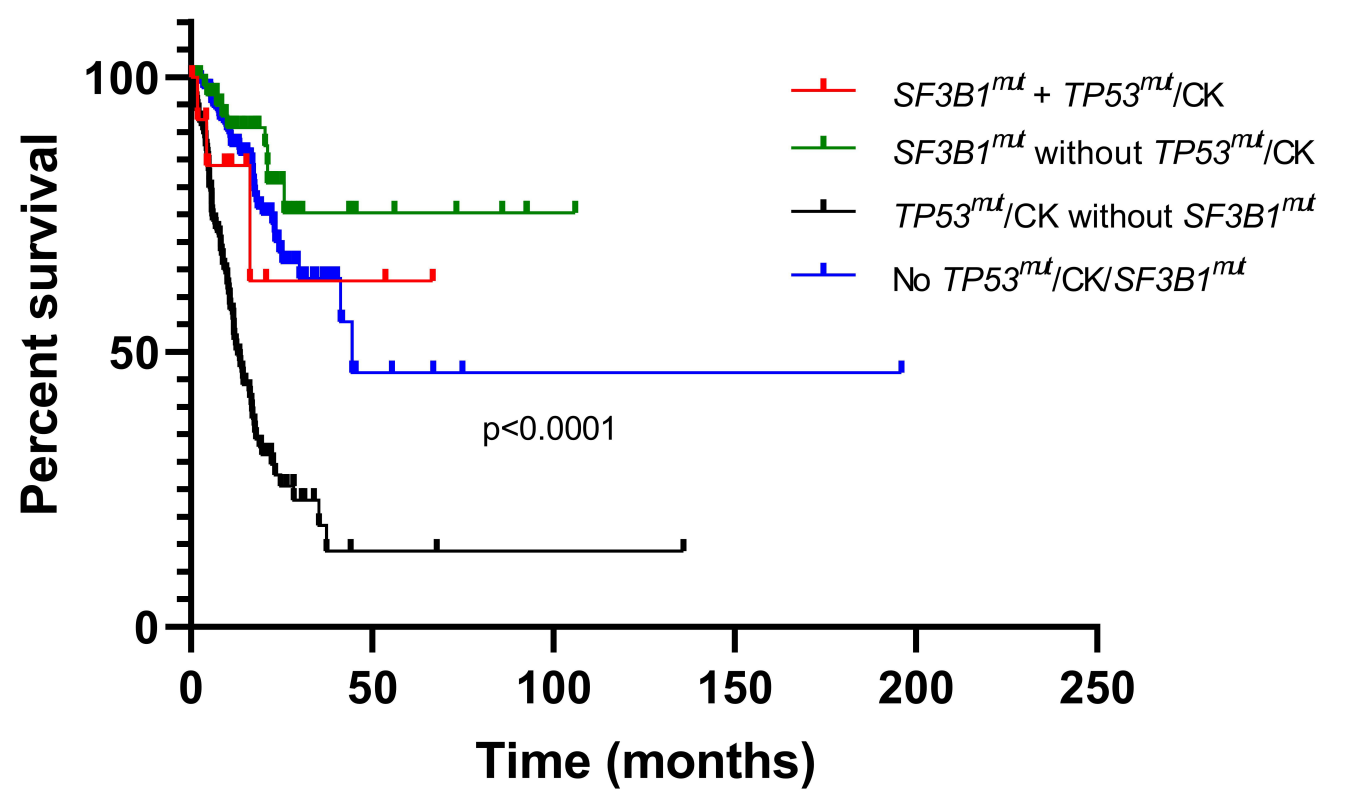

\begin{tabular}{|c|c|c|c|c|c|}
\hline All MDS & Median OS & $\mathbf{n}$ & HR & $95 \% \mathrm{Cl}$ & p \\
\hline$S F 3 B 1^{m u t}+T P 53^{m u t} / C K$ & Undefined & 14 & 1.205 & 0.3356 to 4.329 & 0.7747 \\
\hline SF3B1 ${ }^{\text {mut }}$ without TP53 $3^{m u t} / \mathrm{CK}$ & Undefined & 71 & 0.6035 & 0.3245 to 1.122 & 0.1105 \\
\hline SF3B1wt + TP53 $3^{\text {mut }} / \mathrm{CK}$ & 12.93 & 153 & 4.498 & 3.106 to 6.514 & $<0.0001$ \\
\hline SF3B1wt without TP53 ${ }^{\text {mut }} / \mathrm{CK}$ & 44.47 & 219 & - & - & - \\
\hline
\end{tabular}




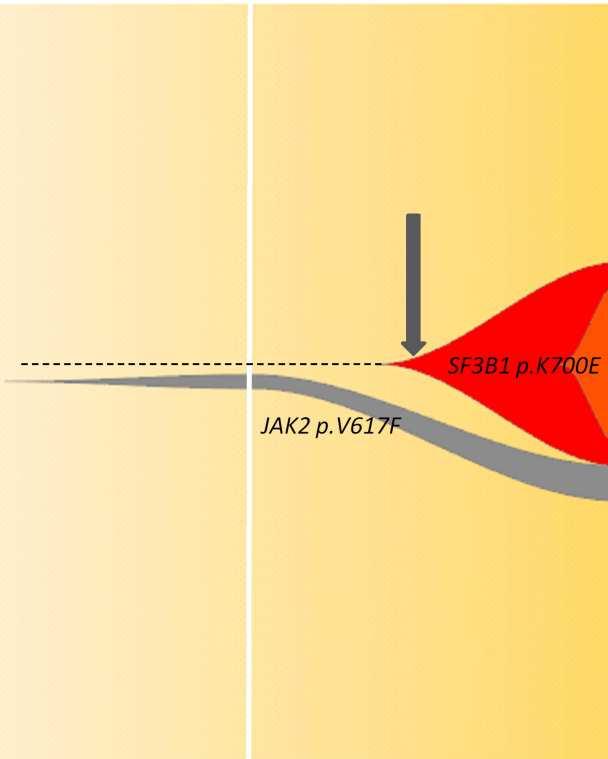

month 0

MDS with $6 \%$ blasts Isolated del(5q)

month 4
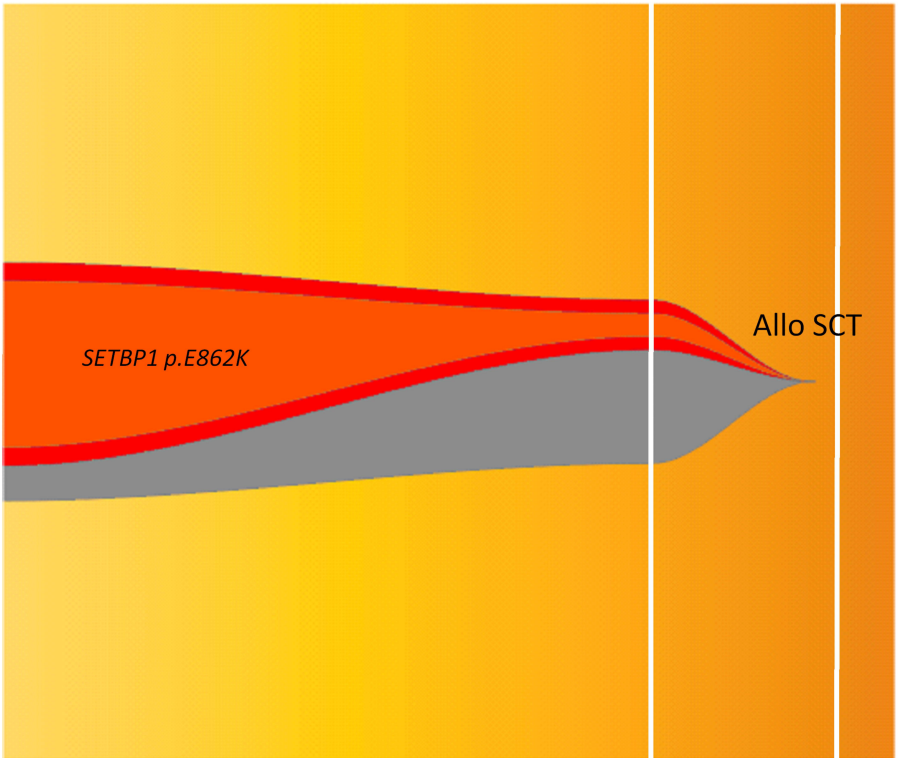

AML with del(5q) month 11

Persistent disease, $\mathbf{5 \%}$ blasts, Normal karyotype month 13

Allo-SCT

B

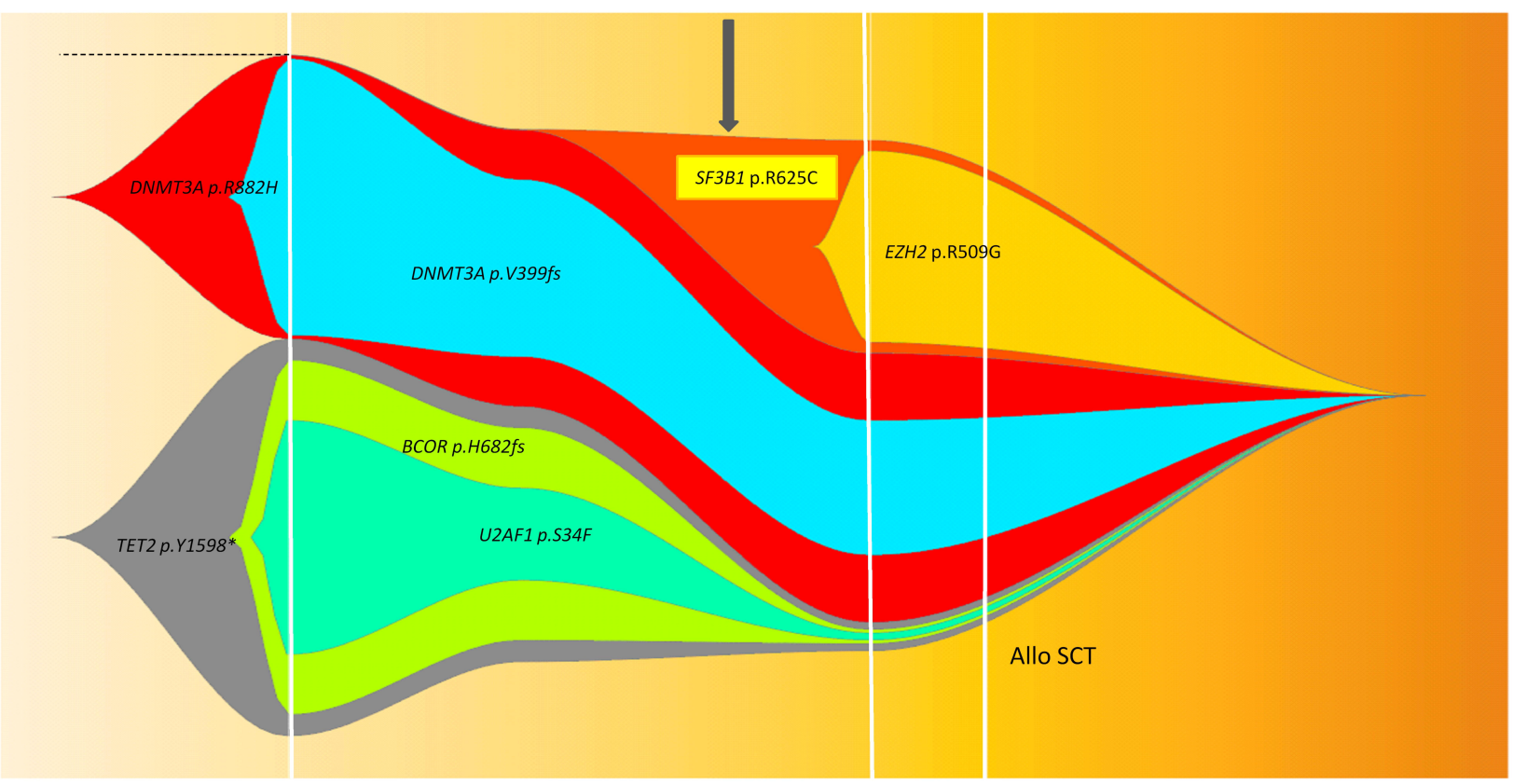

\section{month 0}

MDS with $18 \%$ blasts

$0 \%$ ring sideroblasts

Trisomy 8 month 5

Persistent MDS, $2 \%$ blasts

$18 \%$ ring sideroblasts

Normal karyotype 
bioRxiv preprint doi: https://doi.org/10.1101/2020.09.04.283598; this version posted September 4,2020 . The copyright holder for this preprint (which was not certified by peer review) is the author/funder, who has granted bioRxiv a license to display the preprint in perpetuity. It is made available under aCC-BY-NC-ND 4.0 International license.

Table 1. Baseline characteristics of the study group: all newly diagnosed previously untreated MDS samples that underwent mutation analysis using 81-gene next-generation sequencing panel.

\begin{tabular}{|c|c|c|c|}
\hline Variable & SF3B1 mutated $(n=94)$ & SF3B1 wild-type $(n=415)$ & p-value \\
\hline Age & $74(39-92)$ & $70(23-93)$ & 0.0002 \\
\hline Gender (F:M) & $35: 59$ & $142: 273$ & 0.63 \\
\hline BM blasts\% & $2(0-16)$ & $4(0-19)$ & 0.003 \\
\hline Hemoglobin & $9.2(6.9-12.9)$ & $9.4(5.2-16.4)$ & 0.14 \\
\hline MCV & $105(85-117)$ & $96(66-122)$ & $<0.0001$ \\
\hline Platelet count & $188(8-628)$ & $78(7-692)$ & $<0.0001$ \\
\hline ANC & $2.1(0.2-6.5)$ & $1.3(0-112.9)$ & 0.76 \\
\hline Chemotherapy & $9(9.5 \%)$ & $122(29.4 \%)$ & 0.0001 \\
\hline Radiation & $11(11.7 \%)$ & $79(19.0 \%)$ & 0.10 \\
\hline Therapy-related & $17(18 \%)$ & $142(34 \%)$ & 0.002 \\
\hline Serum EPO & $108(9-1694)$ & $56.9(5.4-5650)$ & 0.70 \\
\hline Serum Ferritin & $462(37-3017)$ & $462(24-7261)$ & 0.47 \\
\hline \multicolumn{4}{|c|}{ Morphologic findings } \\
\hline Ring sideroblasts & $0.4(0-0.9)$ & $0.07(0-0.76)$ & $<0.0001$ \\
\hline \multicolumn{4}{|c|}{ WHO 2016 sub-classification } \\
\hline Isolated del(5q) & $4(4.3 \%)$ & $3(0.7 \%)$ & $<0.0001$ \\
\hline MDS-SLD-RS & $22(23.4 \%)$ & $5(1.2 \%)$ & $<0.0001$ \\
\hline MDS-MLD & $5(5.3 \%)$ & $137(33 \%)$ & $<0.0001$ \\
\hline MDS-MLD-RS & $30(31.9 \%)$ & $28(6.7 \%)$ & $<0.0001$ \\
\hline MDS-EB & $31(33 \%)$ & $201(48.4 \%)$ & $<0.0001$ \\
\hline MDS-U & $2(2.1 \%)$ & $41(9.9 \%)$ & 0.012 \\
\hline \multicolumn{4}{|c|}{ R-IPSS risk categories } \\
\hline Very High & $12(12.8 \%)$ & $107(28.8 \%)$ & 0.0014 \\
\hline High & $9(9.6 \%)$ & $80(21.5 \%)$ & 0.0079 \\
\hline Intermediate & $13(13.8 \%)$ & $75(20.2 \%)$ & 0.19 \\
\hline Low & $37(39.4 \%)$ & $95(25.5 \%)$ & 0.010 \\
\hline Very Low & $14(14.9 \%)$ & $15(4 \%)$ & 0.0004 \\
\hline \multicolumn{4}{|c|}{ IPSS risk categories } \\
\hline Low & $38(45.2 \%)$ & $69(18.6 \%)$ & $<0.0001$ \\
\hline Int-1 & $28(33.3 \%)$ & $174(46.9 \%)$ & 0.028 \\
\hline Int-2 & $16(19 \%)$ & $103(27.8 \%)$ & 0.13 \\
\hline High & $2(2.4 \%)$ & $25(6.7 \%)$ & 0.20 \\
\hline \multicolumn{4}{|c|}{ Karyotype } \\
\hline Diploid & $46(52 \%)$ & $136(35 \%)$ & 0.004 \\
\hline Complex karyotype $(\geq 3)$ & $9(9.6 \%)$ & $133(36 \%)$ & 0.47 \\
\hline
\end{tabular}


bioRxiv preprint doi: https://doi.org/10.1101/2020.09.04.283598; this version posted September 4,2020 . The copyright holder for this preprint (which was not certified by peer review) is the author/funder, who has granted bioRxiv a license to display the preprint in perpetuity. It is made available under aCC-BY-NC-ND 4.0 International license.

Table 2. Comparison of baseline characteristics of all newly diagnosed untreated SF3B1 mutated MDS segregated by the mutation type, K700E vs. non-K700E.

\begin{tabular}{|c|c|c|c|}
\hline Clinical Variable & $\begin{array}{c}\text { SF3B1 K700E } \\
(n=55,59 \%)\end{array}$ & $\begin{array}{l}\text { SF3B1 Non-K700E } \\
(\mathrm{n}=39,41 \%)\end{array}$ & $p$-value \\
\hline Age & $74(39-92)$ & $74(51-86)$ & 0.946 \\
\hline BM blasts $\%$ & $2(0-16)$ & $3(0-16)$ & 0.122 \\
\hline Gender (F:M) & $19 ; 36$ & $16 ; 23$ & \\
\hline Ring sideroblasts & $0.5(0-0.91)$ & $0.34(0-0.77)$ & 0.038 \\
\hline Hemoglobin & $9.35(7-12.9)$ & $9.15(6.9-12.5)$ & 0.675 \\
\hline MCV & $106.5(86-116)$ & $103.5(85-117)$ & 0.248 \\
\hline Platelet count & $195.5(12-628)$ & $123.5(8-421)$ & 0.054 \\
\hline ANC & $2.41(0.48-6.46)$ & $1.78(0.22-4.46)$ & 0.005 \\
\hline Serum EPO & $102(9-1694)$ & $114.4(14.2-1286.4)$ & 0.446 \\
\hline Serum Ferritin & $527(72-3017)$ & $373(37-1725)$ & 0.157 \\
\hline Chemoradiation & $10(18.1 \%)$ & $7(17.9 \%)$ & 1.00 \\
\hline \multicolumn{4}{|c|}{ R-IPSS } \\
\hline Very-high & $5(10 \%)$ & $7(19 \%)$ & 0.226 \\
\hline High & $2(4 \%)$ & $7(19 \%)$ & 0.031 \\
\hline Intermediate & $5(10 \%)$ & $8(22 \%)$ & 0.137 \\
\hline Low & $26(53 \%)$ & $11(31 \%)$ & 0.087 \\
\hline Very low & $11(22 \%)$ & $3(8 \%)$ & 0.143 \\
\hline \multicolumn{4}{|c|}{2016 WHO sub-classification } \\
\hline MDS-SLD-RS, MDS-MLD-RS & $35(64 \%)$ & $17(44 \%)$ & 0.06 \\
\hline MDS-MLD & $3(5 \%)$ & $2(5 \%)$ & 1 \\
\hline MDS-EB & $12(22 \%)$ & $19(49 \%)$ & 0.008 \\
\hline MDS-U & $1(2 \%)$ & 0 & 1 \\
\hline MDS with isolated del(5q) & $4(7 \%)$ & 0 & 0.139 \\
\hline Cytogenetics & $\mathrm{n}=49$ & $\mathrm{n}=36$ & \\
\hline Diploid karyotype & $28(57 \%)$ & $18(50 \%)$ & 1.0 \\
\hline Complex karyotype & $3(6 \%)$ & $6(16.7 \%)$ & 0.159 \\
\hline \multicolumn{4}{|c|}{ Somatic mutations } \\
\hline SF3B1 VAF & $33.5(1.1-46.8)$ & $29.5(1.1-45)$ & 0.18 \\
\hline TET2 & $14(25.5 \%)$ & $9(23.1 \%)$ & 0.691 \\
\hline DNMT3A & $12(21.8 \%)$ & $8(20.5 \%)$ & 0.655 \\
\hline TP53 & $7(12.7 \%)$ & $2(5.1 \%)$ & 0.949 \\
\hline RUNX1 & $4(7.3 \%)$ & $10(25.6 \%)$ & 0.015 \\
\hline$A S X L 1$ & $4(7.3 \%)$ & $3(7.7 \%)$ & 0.618 \\
\hline$E Z H 2$ & $2(3.6 \%)$ & $1(2.6 \%)$ & 0.804 \\
\hline$R A S$ & $2(3.6 \%)$ & $1(2.6 \%)$ & 0.804 \\
\hline FLT3 D835 & $1(1.8 \%)$ & $1(2.6 \%)$ & 0.660 \\
\hline JAK2 & $1(1.8 \%)$ & $1(2.6 \%)$ & 0.660 \\
\hline$B C O R$ & 0 & $4(10.3 \%)$ & 0.027 \\
\hline
\end{tabular}


bioRxiv preprint doi: https://doi.org/10.1101/2020.09.04.283598; this version posted September 4, 2020. The copyright holder for this preprint (which was not certified by peer review) is the author/funder, who has granted bioRxiv a license to display the preprint in perpetuity. It is made available under aCC-BY-NC-ND 4.0 International license.

\begin{tabular}{|l|l|l|l|}
\hline IDH2 & 0 & $3(7.7 \%)$ & 0.068 \\
\hline SRSF2 & 0 & $3(7.7 \%)$ & 0.068 \\
\hline U2AF1 & 0 & $2(5.1 \%)$ & 0.170 \\
\hline
\end{tabular}


bioRxiv preprint doi: https:/doi org/10.1101/2020.09.04 283598: this version posted September 4, 2020. The copyright holder for this preprint (which was not certified by peer review) is the author/funder, who has granted bioRxiv a license to display the preprint in perpetuity. It is made available under aCC-BY-NC-ND 4.0 International license.

Table 3. Univariate analysis for overall survival in all newly diagnosed untreated MDS patients.

\begin{tabular}{|c|c|c|c|}
\hline & $p$-value & OR & $95 \% \mathrm{Cl}$ \\
\hline Age & 0.412 & 1.006 & $0.992-1.020$ \\
\hline Gender & 0.052 & 0.720 & $0.518-1.002$ \\
\hline BM blasts & $<0.001$ & 1.081 & 1.047-1.117 \\
\hline ANC & 0.330 & 1.013 & $0.987-1.041$ \\
\hline Hemoglobin & $<0.001$ & 0.693 & $0.625-0.769$ \\
\hline Platelet count & $<0.001$ & 0.995 & $0.992-0.997$ \\
\hline Mean Corpuscular & $<0.001$ & 0.958 & $0.943-0.974$ \\
\hline Therapy-related & $<0.001$ & 2.134 & $1.539-2.959$ \\
\hline \multicolumn{4}{|l|}{ R-IPSS } \\
\hline Very Low & 0.199 & 0.266 & $0.035-2.011$ \\
\hline Low & - & - & - \\
\hline Intermediate & 0.002 & 2.896 & $1.491-5.627$ \\
\hline High & $<0.001$ & 3.911 & $2.075-7.372$ \\
\hline Very High & $<0.001$ & 10.682 & $6.030-$ \\
\hline Complex karyotype & $<0.001$ & 4.910 & $3.483-6.920$ \\
\hline MDS-EB & $<0.001$ & 2.167 & $1.494-3.144$ \\
\hline \multicolumn{4}{|l|}{ SF3B1 } \\
\hline Wild type & - & - & - \\
\hline K700E & 0.001 & 0.175 & $0.064-0.473$ \\
\hline Non-K700E & 0.224 & 0.657 & $0.334-1.293$ \\
\hline SF3B1 VAF & 0.026 & 0.959 & $0.924-0.995$ \\
\hline \multicolumn{4}{|c|}{ NGS mutations (seen in $>5 \%$ of cases) } \\
\hline$A S X L 1$ & 0.817 & 0.950 & $0.612-1.473$ \\
\hline$B C O R$ & 0.114 & 0.324 & $0.080-1.309$ \\
\hline DNMT3A & 0.650 & 0.896 & $0.558-1.438$ \\
\hline IDH1/IDH2 & 0.127 & 0.498 & $0.204-1.218$ \\
\hline$R A S$ & 0.434 & 0.700 & $0.287-1.709$ \\
\hline$R U N X 1$ & 0.760 & 0.920 & $0.539-1.570$ \\
\hline SRSF2 & 0.499 & 0.832 & $0.487-1.420$ \\
\hline TET2 & 0.002 & 0.469 & $0.289-0.760$ \\
\hline TP53 & $<0.001$ & 5.158 & $3.699-7.193$ \\
\hline U2AF1 & 0.023 & 0.437 & $0.214-0.891$ \\
\hline
\end{tabular}


bioRxiv preprint doi: https://doi.org/10.1101/2020.09.04.283598; this version posted September 4, 2020. The copyright holder for this preprint (which was not certified by peer review) is the author/funder, who has granted bioRxiv a license to display the preprint in perpetuity. It is made available under aCC-BY-NC-ND 4.0 International license.

Table 4. Multivariate analysis for overall survival in all newly diagnosed untreated MDS patients ( $p$-value cutoff of 0.200 from univariate analysis) SF3B1 mutations were segregated by subtype.

\begin{tabular}{|l|c|c|c|}
\hline & p-value & OR & 95\% Cl \\
\hline Hemoglobin & $<0.001$ & 0.805 & $0.713-0.909$ \\
\hline \multicolumn{5}{|l|}{ R-IPSS } \\
\hline Very Low & 0.282 & 0.326 & $0.042-2.512$ \\
\hline Low & - & - & - \\
\hline \multicolumn{4}{|l|}{} \\
\hline High & 0.016 & 2.288 & $1.166-4.487$ \\
\hline Very High & 0.010 & 2.436 & $1.237-4.798$ \\
\hline SF3B1 & $<0.001$ & 4.143 & $2.076-8.467$ \\
\hline Wild type & - & - & - \\
\hline K700E & 0.041 & 0.288 & $0.087-0.951$ \\
\hline Non- & 0.804 & 0.913 & $0.445-1.873$ \\
\hline BCOR & 0.154 & 0.234 & $0.032-1.724$ \\
\hline TP53 & $<0.001$ & 2.374 & $1.539-3.662$ \\
\hline U2AF1 & 0.082 & 0.513 & $0.242-1.089$ \\
\hline
\end{tabular}

